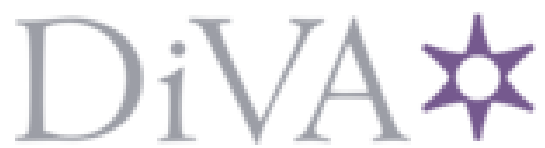

http://www.diva-portal.org

This is the published version of a paper published in Physical Chemistry, Chemical Physics $P C C P$.

Citation for the original published paper (version of record):

Claesson, P M., Dobryden, I., Li, G., He, Y., Huang, H. et al. (2017)

From force curves to surface nanomechanical properties

Physical Chemistry, Chemical Physics - PCCP, 19(35): 23642-23657

https://doi.org/10.1039/c7cpo2612a

Access to the published version may require subscription.

N.B. When citing this work, cite the original published paper.

Permanent link to this version:

http://urn.kb.se/resolve?urn=urn:nbn:se:kth:diva-215359 
Check for updates

Cite this: Phys. Chem. Chem. Phys., 2017, 19, 23642

Received 20th April 2017, Accepted 22nd May 2017 DOI: $10.1039 / c 7 c p 02612 a$ rsc.li/pccp

\title{
From force curves to surface nanomechanical properties
}

\author{
Per M. Claesson, (D) *ab Illia Dobryden, ${ }^{a}$ Gen Li, ${ }^{a}$ Yunjuan He, ${ }^{a}$ Hui Huang, ${ }^{a}$ \\ Per-Anders Thorén ${ }^{c}$ and David B. Haviland ${ }^{c}$
}

\begin{abstract}
Surface science, which spans the fields of chemistry, physics, biology and materials science, requires information to be obtained on the local properties and property variations across a surface. This has resulted in the development of different scanning probe methods that allow the measurement of local chemical composition and local electrical and mechanical properties. These techniques have led to rapid advancement in fundamental science with applications in areas such as composite materials, corrosion protection and wear resistance. In this perspective article, we focussed on the branch of scanning probe methods that allows the determination of surface nanomechanical properties. We discussed some different AFM-based modes that were used for these measurements and provided illustrative examples of the type of information that could be obtained. We also discussed some of the difficulties encountered during such studies.
\end{abstract}

\section{Introduction}

Studies of the interactions between surfaces date back to ancient times, where one aim was to allow solid bodies to slide against each other without the need to apply a very high force. The understanding of this subject was summarized by Leonardo da Vinci and Amontons and others in medieval times. These studies form the basis for what now is known as Amontons' rules of friction, which states that the friction force is proportional to the load, independent of contact area and independent of shear velocity. Although these rules are often described as laws, there is no fundamental principle that forbids a system to display friction properties that do not agree with these rules. ${ }^{1}$ Another ancient issue is the stability of colloidal particles in liquid media, where for a long time it has been known that some dispersions are stable and others flocculate, and that the dispersion stability can be affected by the addition of salts, surface active or polymeric additives. The stability is related to the forces acting between the particles in the liquid medium, and since the nature of the particle surface is of paramount importance for determining these interactions they are now commonly discussed using the term surface forces. ${ }^{2}$

\footnotetext{
${ }^{a}$ KTH Royal Institute of Technology, School of Chemical Science and Engineering, Department of Chemistry, Surface and Corrosion Science, Drottning Kristinas väg 51, SE-100 44 Stockholm, Sweden. E-mail: percl@kth.se

${ }^{b}$ SP Technical Research Institute of Sweden, SP Chemistry, Materials and Surfaces, Box 5607, SE-114 86 Stockholm, Sweden

${ }^{c}$ KTH Royal Institute of Technology, Nanostructure Physics, Roslagstullsbacken 21, SE-106 91 Stockholm, Sweden
}

Theoretical understanding of surface forces was achieved by several pioneering efforts. Special mention should be given to Derjaguin and Landau ${ }^{3}$ as well as Verwey and Overbeek ${ }^{4}$ who independently and simultaneously formulated the DLVO theory which discusses colloidal stability in terms of electrical doublelayer forces and van der Waals forces. A better understanding of the nature of the van der Waals forces between macroscopic surfaces was later provided by Lifshitz and co-workers. ${ }^{5} \mathrm{~A}$ recent comprehensive textbook on this topic has been written by Parsegian. ${ }^{6}$ The understanding of surface forces generated by adsorbed polymer layers came later due to the efforts of many scientists, of whom special mention should be given to deGennes ${ }^{7}$ and Fleer, Cohen-Stuart, Scheutjens, Cosgrove and Vincent ${ }^{8}$ who wrote classical textbooks on this topic. A general and informative book on surface forces is provided by Israelachvili, which is recommended for anyone interested in this subject. ${ }^{2}$

Of course, theory cannot be developed without advancement in experimental methodologies. When it became possible to measure surface forces and to critically test theoretical predictions, evidence was found for other types of surface forces and this field developed rapidly. Measurement of the distance dependence of the weak long-range forces that govern colloidal stability required large surfaces, for instance in the surface forces apparatus (SFA), semi-cylindrical surfaces with a radius of curvature of about $2 \mathrm{~cm}$ are used. ${ }^{9}$ As the detection sensitivity for these forces increased, smaller surfaces could be used, for example in the AFM colloidal probe method ${ }^{10}$ the interaction between a particle with a radius of a few micrometres and a flat surface is commonly studied. 
When measuring the strong forces that act between two surfaces in close proximity, one can use a probe with an even smaller radius. The atomic force microscope (AFM), which was initially used for topographical imaging, uses a sharp tip with an end-radius in the nm range, where, using the contact mode, one creates a topographic image by noting the position of the piezo actuator that carries the surface at a given applied load (i.e. constant cantilever deflection). ${ }^{11}$ Alternatively, in tapping mode, the amplitude of the oscillation is kept constant as the tip scans over the surface. ${ }^{12}$ The tapping mode does not only produce a topography image, but also a phase image that reflects the changes in the phase-lag between the freely oscillating cantilever and that experienced when the tip is tapping on the surface. The phase image is affected by material properties, such as tip-sample adhesion and viscoelasticity of the sample surface. ${ }^{13}$ In fact, as the tip oscillates in and out of contact with the surface it is affected by separation-dependent surface forces as well as surface viscoelasticity when the tip deforms the surface and penetrates into it. Nowadays, with high-speed data capturing it is possible to record complete force curves at each image pixel. It is from such force curves that one extracts local surface nanomechanical properties, either directly from the force curve or by fitting models from contact mechanics to parts of the measured force curves. The most common models used for this purpose are due to the work by Hertz, ${ }^{14}$ Derjaguin Müller and Toporov (DMT model), ${ }^{15}$ Johnson Kendall and Roberts (JKR model) ${ }^{16}$ and Sneddon. ${ }^{17}$ Before discussing some of the AFM-based methods used for surface nanomechanical mapping, we briefly discuss some of the classical indentation methods that are used for the determination of mechanical properties.

\section{Indentation methods}

Throughout the history of materials science there has been a need to measure the hardness of different materials. One set of methods used for such measurements are based on indenting a small object into the material of interest. Here, we mention two of these methods, microindentation and nanoindentation which differ in the load applied during measurements. We also generally compare the AFM-based methods with these two methods. A detailed review on this topic is provided by Drelich et al. ${ }^{18}$

\section{Microindentation}

The first static hardness test employing a microindenter, a $10 \mathrm{~mm}$ hardened steel ball, was introduced in 1900 by the Swedish researcher J. A. Brinell, ${ }^{19}$ which was limited by the material properties of the metal indenter and not suitable for hard materials. The reason for this is that the metal indenter sphere could deform during the test, which could lead to significant error in the evaluated hardness. To overcome this problem a hard spherical diamond indenter was introduced by Shore. ${ }^{20}$ However, the manufacture of a perfectly hemispherical diamond surface was a key obstacle. All these limitations were overcome in a new method, the Vickers hardness test, introduced by Smith and Sandland in $1922 .^{21}$ This method utilizes a square-based diamond pyramid indenter, which allows the hardness of basically any material to be evaluated, except diamond. The Vickers test remains one of the most common methods even today, and it has been successfully applied to evaluate material hardness of a broad range of materials. However, the Vickers test is not very suitable for small specimens, brittle materials and thin films. For these reasons Knoop introduced an alternative hardness test in $1939,{ }^{22}$ which employed a rhombohedral-shaped diamond indenter at low-loads. A more detailed overview of the historical origins of indentation hardness testing can be found in the article by Walley. ${ }^{23}$ Thus, it can be concluded that the microindenter methods for studying the bulk mechanical properties of various materials are well-developed, despite several known problems, for instance the indentation size effect phenomenon. ${ }^{24}$ Further, the main limitation of these methods, which is dictated by the rapid development of nanotechnology, is that they are not suitable for exploring material mechanical properties at the nanoscale.

\section{Nanoindentation}

The need for the accurate characterization of mechanical properties of thin films with nanoscale thickness led to the successful development of ultra-low load indentation techniques. Pharr and Oliver proposed such a method in 1992 in order to measure hardness and elastic modulus by determining them directly from indentation load $v$. displacement data. ${ }^{25,26}$ In this technique a sharp Berkovich triangular pyramid, or alternatively a sphere, is used as the indenter ${ }^{27}$ to resolve changes in indentation depth at the nanometre level. This method is suitable for thin films of less than a few hundreds of $\mathrm{nm}$ and follows the rule that the depth of the contact area has to be less than $10 \%$ of the film thickness. The method proposed by Pharr and Oliver is now routinely used in various nanoindenter systems, and it has been successfully applied to measure the nanomechanical properties of various materials. ${ }^{28}$ However, several aspects may contribute to measurement errors, such as substrate stiffness contribution, crystalline anisotropy, effective indenter shape and pile-up effects. Some of these errors can be corrected as proposed by Pharr $^{28}$ and Menchik. ${ }^{29}$ We note that even though the depth of the imprints is on the nanoscale, their lateral size is often on the $\mu \mathrm{m}$ scale or at least hundreds of $\mathrm{nm}$, thus the size of the contact area limits the ability to probe nanomechanical properties on ultra-small areas on the surface. Further, the study of soft matter materials, such as gels and biomaterials, require an even gentler technique. ${ }^{30}$

\section{AFM-based methods}

The pioneering work using AFM as a tool to probe nanomechanical properties was reported by Colton and Burham in $1989 .{ }^{31} \mathrm{~A}$ few years later Brushan and Kionkar demonstrated that AFM nanoindentation not only has the important advantage of high spatial resolution, but also can be used with very small $(\approx \mathrm{nm})$ indentation depths. ${ }^{32}$ However, a known problem is the cantilevermounting angle, which causes a small lateral motion of the tip during indentation. ${ }^{33}$ Since its introduction, the AFM nanoindentation technique has been successfully applied to various types of surfaces, and it is not limited to soft matter and 
biological samples since the nanomechanical properties of hard surfaces have also been determined successfully. ${ }^{33} \mathrm{~A}$ recent review on this topic is provided by Tsukruk et al. ${ }^{34}$ Another valuable review by Tsukruk et al. focuses on probing soft matter using AFM methods. ${ }^{35}$ A contact mechanics model needs to be applied in order to connect the AFM nanoindentation measurements to nanomechanical properties. Such models were introduced and widely used at high loads long before the AFM technique was invented, but then under conditions where the contact forces were larger than the surfaces forces. The Hertzian model introduced in the end of the 19th century ${ }^{14}$ was later extended to take into account the short-range attractive forces within the region of contact, which is known as the Johnson, Kendall, and Roberts (JKR) model. ${ }^{16}$ Another commonly used model takes into account the long-range attractive forces acting outside the region of contact, which is known as the Derjaguin, Muller, and Toporov (DMT) model. ${ }^{15}$ Further, a model accounting for viscoelastic deformation in the transition regime between the JKR and DMT models was developed by Maugis and GauthierManuel. ${ }^{36}$ Often, instead of Hertzian contact mechanics between a sphere and flat surface, which does not account for the actual tip geometry and inelastic deformation, Sneddon mechanics is used. ${ }^{17,37}$

The most widely used single frequency dynamic AFM technique is the tapping mode (TM) or amplitude modulation atomic force microscopy. ${ }^{38}$ This technique provides some information on surface mechanical properties via a phase image which reports the oscillation phase difference between the free response of the cantilever (i.e. when not interacting with the surface) and when engaged with the surface. This image shows a contrast due to changes in material properties, such as surface viscoelasticity and tip-sample adhesion. ${ }^{39}$ The value of the phase shift is also influenced by experimental parameters such as set point and tapping frequency, which complexly depend on the tip-surface interaction and probe height. ${ }^{40,41}$ In this mode the force $v s$. tip position curve is not captured, which complicates further analysis. However, a report describes force reconstruction from tapping mode experiments. ${ }^{42}$

Single frequency AFM methods, such as Peak Force Tapping (PeakForce QNM) ${ }^{43,44}$ quantitative imaging, QI, ${ }^{45}$ force volume mapping ${ }^{46}$ and other modes, ${ }^{47}$ allow capturing of the complete force curve. Outstanding force control facilitates the maintenance and minimization of the peak force or interaction force down to tens of pico Newtons. These modes have the common feature that the operator decides the maximum force applied, but they differ in terms of the oscillation frequency used and how the captured force curves are analysed to provide nanomechanical information related to the surface under investigation. An example of this is provided in Fig. 1, which illustrates how the captured force curve is analysed in Peak Force Tapping. In this mode several images are obtained simultaneously. The surface topography is shown in one image and the other images are reconstructed from the force curves. The adhesion image is extracted from the retraction force curve and displays the maximum attractive force experienced upon separation. The deformation image shows how much the surface has been compressed at the

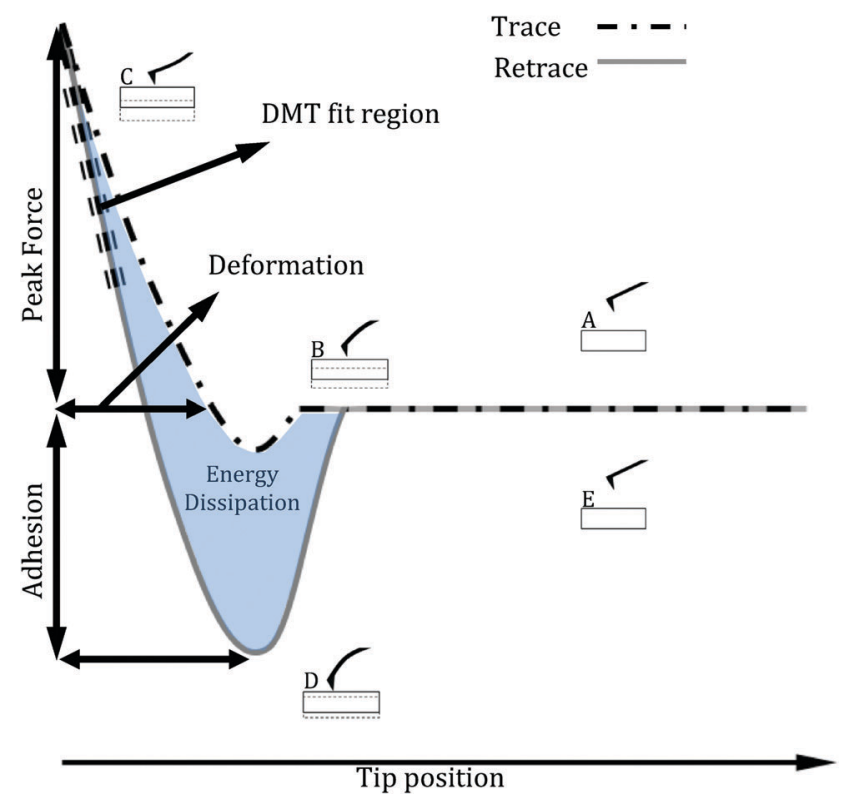

Fig. 1 Schematic of one cycle of the force-separation curve in peak force tapping. Surface mechanical properties such as deformation, adhesion, energy dissipation, and elastic modulus are measured at each pixel in the image. Reprinted with permission from ref. 48. Copyright, 2017 American Chemical Society. At large separations the cantilever is unaffected by the surface (A), it bends towards the surface under attractive forces (B) and away from the surface under repulsive forces (C). Upon separation a maximum attraction is encountered (D) before the tip again detaches from the surface (E).

maximum applied force (the peak force), and the energy dissipation image is the integrated hysteresis between the trace and retrace force curves (shaded area in Fig. 1). The elastic modulus of the sample surface is extracted by fitting, for example, the DMT contact mechanics model to a part of the retrace force curve. Of these quantities the elastic modulus is most easily compared to the quantities determined by other methods such as indentation methods but it is also the least reliable quantity. The reason for this is that it requires fitting of a contact mechanics model to the measurements, and the chosen contact mechanics model may not be fully appropriate for the particular surface under investigation. It should also be noted that the different quantities extracted from such force curves are not completely independent, as will be discussed in the cross-talk section.

Recent developments in the study of surface nanomechanical properties include the use of multi-frequency dynamic AFM methods. ${ }^{4-52}$ Such approaches provide more information on the surface nanomechanical response, but are less intuitive since they do not directly give a force vs. tip position curve, which is commonly used to understand the tip-surface interaction in AFM. However, different types of force curves can be reconstructed from the multi-frequency probing of the sample. In this article we discuss one such multi-frequency method known as Intermodulation AFM (ImAFM). ${ }^{53}$

However, it goes beyond the scope of this article to describe the details of the mathematical treatment that underlines the evaluation of ImAFM data, which can be found in the literature. ${ }^{54}$ 
Rather, we focus on the essential features of this technique where the cantilever is simultaneously excited with two tones at different frequencies close to the resonance frequency of the cantilever. For example in one of our measurements the resonance frequency was $313.9 \mathrm{kHz}$ and the two drive tones were placed at frequencies of $f_{1}=313.7 \mathrm{kHz}$ and $f_{2}=314.2 \mathrm{kHz}$, roughly centred on resonance, and spaced by $500 \mathrm{~Hz}$. As the cantilever motion is affected by the non-linear tip-surface interaction, new tones appear in the response near resonance. These new tones are known as intermodulation (frequency mixing) products, which explains the name Intermodulation AFM. These intermodulation products occur at specific values of frequency given by:

$$
f_{\mathrm{IMP}}=m f_{1}+n f_{2}
$$

where, $m$ and $n$ are integers (positive or negative) and $|n|+|m|$ is the order of the intermodulation product, ${ }^{55}$ which carry information about the nonlinear character of the tip-surface interaction and from them we can extract the surface nanomechanical properties. ${ }^{56,57}$ A multi-frequency lock-in amplifier measures the amplitude and phase of each IMP at every image pixel while scanning.

Due to the fact that the cantilever used in ImAFM measurements has a high $\mathrm{Q}$ resonance, the multifrequency response is crowded in a relatively narrow frequency band around resonance, and in the time domain, it describes the slow amplitude modulation of a fast oscillation at the resonant frequency. In this case the multi-frequency intermodulation response can be directly transformed into two force-quadrature curves which represent the integrals of the tip-surface force over each single fast oscillation cycle of amplitude $A$. One curve, $F_{\mathrm{I}}(A)$, gives the integrated force in-phase with the cantilever oscillation and the other $F_{\mathrm{Q}}(A)$ gives the integrated force quadrature to the oscillation or in-phase with the velocity. This subdivision into in-phase and quadrature components is analogous to the data treatment of the rheological response determined using oscillatory shear measurements and the electrical response obtained using impedance spectroscopy. $F_{\mathrm{I}}(A)$ and $F_{\mathrm{Q}}(A)$ are essentially Fourier coefficients of the force, or time integrated quantities, but are represented in a special way such that they are in-phase and quadrature with respect to the cantilever motion, respectively. ${ }^{54}$ These quantities are calculated as:

$$
\begin{gathered}
F_{\mathrm{I}}=\frac{1}{T} \int_{0}^{T} F(t) \cos (\varpi t) \mathrm{d} t \\
F_{\mathrm{Q}}=\frac{1}{T} \int_{0}^{T} F(t) \sin (\varpi t) \mathrm{d} t \\
z(t)=h+A \cos (\varpi t) .
\end{gathered}
$$

where, $h$ is the working distance, i.e. the probe height or separation between the tip and surface when both are in the equilibrium position, and $z(t)$ is the tip position. The integral goes over one oscillation cycle $(T \approx 3 \mu \mathrm{s})$. Hundreds of oscillation cycles are carried out at each pixel where the amplitude first slowly increases with each cycle up to its maximum value (typically about $100 \mathrm{~nm}$ ), and then decreases back to zero.

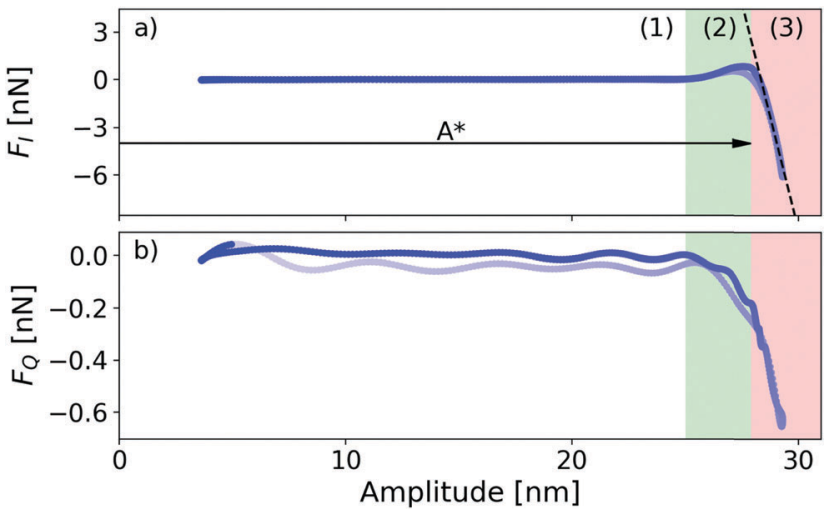

Fig. 2 Typical (a) $F_{1}(A)$ and (b) $F_{Q}(A)$ quadrature curves reconstructed on a polished steel surface. The force quadratures on stiff materials can generally be split into three regions: (1) marked in white, no interaction, (2) dominantly attractive interaction, marked in green, and (3) dominantly repulsive interaction, marked in red. In the dominantly repulsive region of the $F_{1}(A)$ curve a line can be fitted, which is marked with dashes in the figure. The light blue curve is measured as the amplitude increases, whereas the dark blue is measured as the amplitude decreases again. The slope of this line defines the $F_{1}$-stiffness, a quantity related to the stiffness, or elasticity, of the surface. The amplitude marked by $A^{*}$ is the amplitude that gives rise to maximum attraction in the $F_{1}(A)$ curve.

This amplitude modulation sequence takes two milli-seconds to complete, thus providing the amplitude dependence of $F_{\mathrm{I}}(A)$ and $F_{\mathrm{Q}}(A)$ at each image pixel. An example of such data is shown in Fig. 2.

The functions $F_{\mathrm{I}}(A)$ and $F_{\mathrm{Q}}(A)$ may look similar to a traditional force curve, as illustrated in Fig. 1, but they are distinctly different. First, being integrals of force over one cycle, they do not give the force at a given fixed position (as in Fig. 1). Second, they are not plotted as a function of position but rather as a function of the oscillation amplitude. Third, the convention in normal force measurements is that attractive forces have a negative sign, whereas with force quadrature curves, a positive $F_{\text {I }}$ corresponds to a dominantly attractive force and a negative value corresponds to a dominantly repulsive force over a single oscillation cycle where the tip can experience both attractive and repulsive forces during the cycle. We note that the conservative part of the tip-surface interaction, $F_{\mathrm{I}}(A)$, can be converted to a traditional force $v s$. tip position curve (without invoking a model) using the inverse Abel transform. ${ }^{54}$ However, this is not a necessary step for extracting surface nanomechanical data.

When the measurement starts, a small oscillation amplitude is used (below $10 \mathrm{~nm}$ in Fig. 2). At this stage there is no interaction between the tip and the surface and both $F_{\mathrm{I}}$ and $F_{\mathrm{Q}}$ are zero. As the amplitude increases the tip comes closer and closer to the surface at the lower turning point of the oscillation. At some amplitude it will experience an attractive van der Waals force, as shown in region (2) in Fig. 2. Thus the time averaged in-phase force, $F_{1}$, becomes net positive (attractive). If the surface deforms due to this attractive interaction, a dissipative force may arise due to the viscous nature of the surface, giving $F_{\mathrm{Q}}$ with a negative sign. The maximum time integrated force $F_{\mathrm{I}}$ is encountered at the amplitude marked $A^{*}$ in Fig. 2. A further increase 
in amplitude causes the tip to compress and possibly penetrate into the surface before it is retracted, such that the time averaged in-phase force becomes less attractive and eventually repulsive. At the same time the quadrature force $F_{\mathrm{Q}}$ increases in magnitude as more energy is dissipated during the oscillation cycle.

In the next sections we will discuss some systems that have been studied using AFM-based techniques to extract local surface nanomechanical information. Of course, the first question that has to be asked is how local is local?

\section{Single core-shell nanoparticles}

The nanomechanical properties of polymer brush layers on high curvature silica particles $(\approx 10 \mathrm{~nm}$ radius) were probed by Peak Force Tapping. ${ }^{58}$ The particles carried a grafted polymer layer of the weak cationic polyelectrolyte poly(2-(dimethylamino)ethyl methacrylate) (PDMAEMA) with a graft density of 0.43 chains per $\mathrm{nm}^{2}$. The nanoparticles were thus of the core-shell type. The tip qualification procedure in the NanoScope Analysis Software (v. 1.20) from Bruker was used to create a tip image using a titanium roughness sample containing sharp edges (model number RS-15M, Bruker).

The cationic particles were electrostatically adsorbed on flat negatively charged silica surfaces, and the sample surface was then probed by the tip, as illustrated in Fig. 3. The topographical image shown in Fig. 3 visualizes the particles as featureless bright spots. However, the deformation image, which is also presented in Fig. 3, is more interesting since here each particle appears doughnut shaped with a rim that is significantly more deformable than the center of the particle. Similar features are observed in the dissipation and adhesion images (not shown). Since each particle in these images has a similar nanomechanical response we can zoom in on a single particle to gain further insight.

The image of one single particle is illustrated in Fig. 4. The height image suggests that the particle extends above the silica surface by about $15 \mathrm{~nm}$. However, since the image was obtained using the relatively high peak force of $6 \mathrm{nN}$, the height is likely underestimated. The deformation, adhesion and dissipation images all show doughnut shaped particles with higher values encountered at the edge of the particle than on top of it. As discussed in the original article ${ }^{58}$ the asymmetry observed in some of the images is likely an artefact induced by the horizontal motion of the tip during scanning. By azimuthally averaging the properties at a defined distance away from the particle centre (Fig. 4, right) the variation of their values with respect to the distance from the particle centre can be evaluated. The peak values of the deformation, adhesion and dissipation are found in the range of 11-17 $\mathrm{nm}$ from the particle centre. Referring to Fig. 3A, we can see that this is related to how the tip meets the grafted polymer chains. At the centre of the particle the approaching tip will preferentially compress the chains, whereas at the edge of the particle the tip will mostly bend the polymer chains.
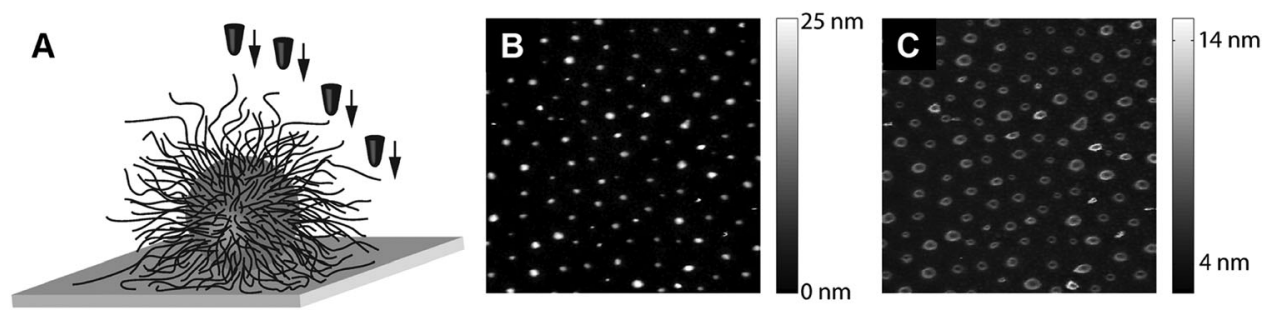

Fig. 3 Nanomechanical mapping of adsorbed brush-grafted nanoparticles. (A) Schematic drawing of the tip probing the brush-grafted nanoparticle by vertical motion at different horizontal positions relative to the particle centre. Overview of multiple adsorbed nanoparticles showing (B), height and (C), deformation. The nanoparticles were adsorbed and imaged at $23^{\circ} \mathrm{C}$ in $1 \mathrm{mM} \mathrm{NaCl}$ at pH 9.2, and the area of the overview is $1.1 \mu \mathrm{m} \times 1.1 \mu \mathrm{m}$. Reproduced and adopted from ref. 58 with permission from the Royal Society of Chemistry.
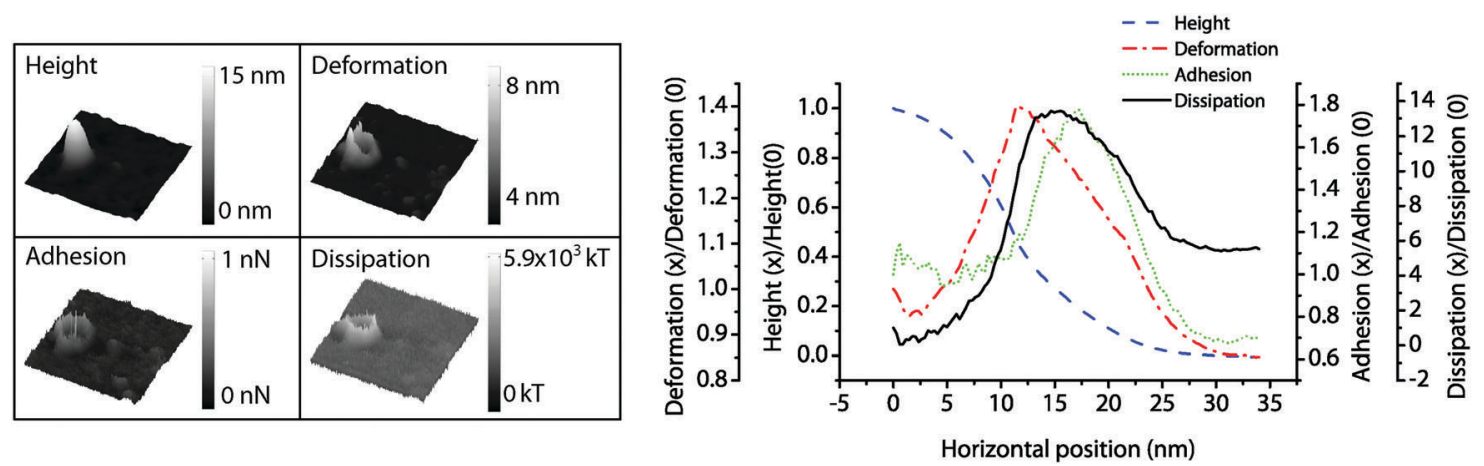

Fig. 4 Left panel: Height, deformation, adhesion and dissipation images of a single Si-PDMAEMA nanoparticle mapped at a peak force of $6 \mathrm{nN}$. The right panel illustrates the azimuthally averaged properties as a function of horizontal distance from the particle centre. The nanoparticle was adsorbed at $\mathrm{pH} 9.2$ in $1 \mathrm{mM} \mathrm{NaCl}$ at $23^{\circ} \mathrm{C}$. Each image is $153 \times 153 \mathrm{~nm}$. Reproduced from ref. 58 with permission from the Royal Society of Chemistry. 
Clearly, it requires less force to bend the polymer chains than to compress them, as seen by the larger deformation at the particle edge. The larger deformation, which suggests further penetration of the tip into the brush layer, gives rise to a larger contact area between the tip and the polymer layer and this is the origin of the higher adhesion force at the edge of the particle. Since the adhesion and dissipation values are coupled (see Fig. 1), the larger dissipation observed at the edge of the particle is to a large extent related to the higher adhesion at this position. However, under some conditions the adhesion between the tip and the polymer layer was found to be very low and still a contrast was observed in the dissipation image. Thus, it appears that more energy is dissipated when the polymer chains are bent compared to when they are compressed.

From these experiments it is clear that different mechanical responses can be recorded across a single nanoparticle with resolution in the nanometre range. These data were acquired on a very smooth substrate surface, thus it is valid to explore if contrast in surface nanomechanical properties can also be achieved on significantly rougher surfaces.

\section{Polymer coatings}

The effect of curing time on the surface nanomechanical properties of a polymer coating was investigated using AFM Peak Force Tapping. The coating was applied on polished coldrolled carbon steel ultrasonically cleaned in absolute ethanol. The applied water-based paint consisted of a high molecular weight acrylic polyester with a melamine cross linker, supplied by PTE Coatings AB (Västervik, Sweden). A homogeneous thin film with a wet thickness of $60 \mu \mathrm{m}$ was deposited using an applicator. The coated samples were pre-dried at room temperature and then exposed to $180{ }^{\circ} \mathrm{C}$ for $2 \mathrm{~min}, 10 \mathrm{~min}$ or $30 \mathrm{~min}$ to cure. The surface topography and elastic modulus of these samples are shown in Fig. 5. The measurements were carried out using silicon Tap300DLC probes (BudgetSensors) with a calibrated end tip radius of $9 \mathrm{~nm}$, as measured on a polycrystalline titanium roughness sample and determined with the tip quantification tool (NanoScope Analysis software). The measured spring constant was $29.8 \mathrm{~N} \mathrm{~m}^{-1}$ and the peak force setpoint was in all cases $50 \mathrm{nN}$ to give sufficient deformation to evaluate the nanomechanical parameters.

The surface of the polymer coating clearly became stiffer with an increase in curing time, and the surface elastic modulus (Table 1) increased by a factor of 3-4 as the curing time was prolonged from $2 \mathrm{~min}$ to $30 \mathrm{~min}$. The average roughness, $R_{\mathrm{a}}$, parameter was hardly affected by the curing temperature. This simple example illustrates that AFM-based measurements provide valuable information on how different processing parameters affect the surface state of polymer coatings. Composite coatings present significantly more heterogeneous surfaces, and in the following section we will illustrate what we

Table 1 Average roughness and elastic modulus evaluated using the standard NanoScope analysis software. Note that the sample cured for 30 minutes was analyzed using a higher peak force

\begin{tabular}{lll}
\hline Curing time, $\min$ & $R_{\mathrm{a}}, \mathrm{nm}$ & Elastic modulus, GPa \\
\hline 2 & 1.15 & 0.23 \\
10 & 1.10 & 0.83 \\
30 & 1.35 & 0.90
\end{tabular}

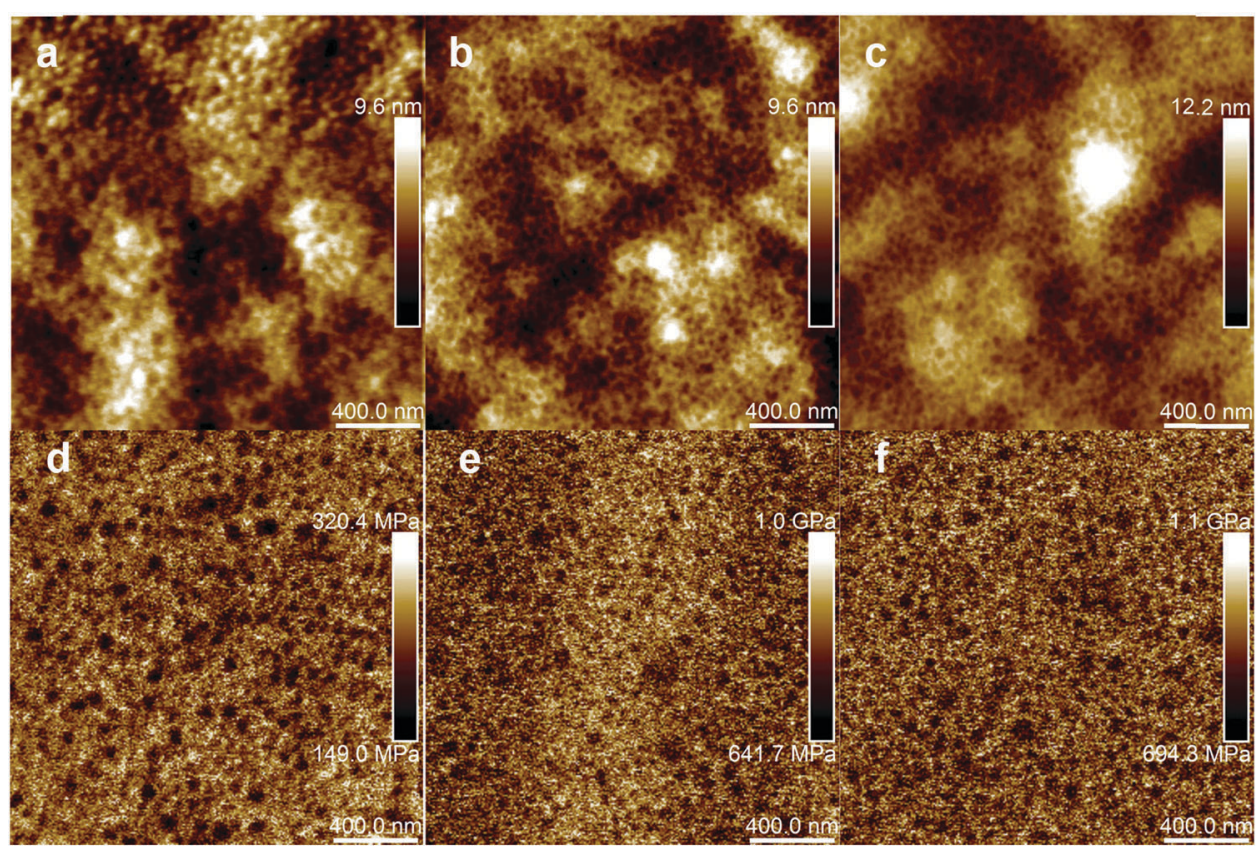

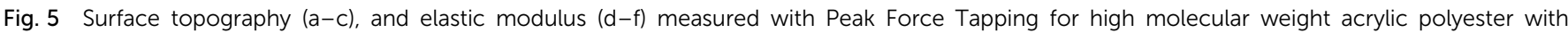

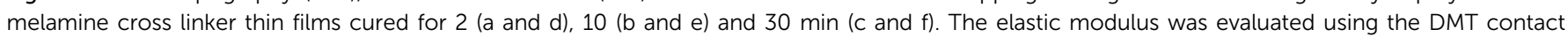
mechanics model and a tip with an evaluated end radius of $9 \mathrm{~nm}$. 
can learn from AFM-based nanomechanical mapping techniques for such systems.

\section{Macrocomposites}

Fiber-based materials are widely used for packaging, and in order to protect contents against physical and chemical damage a coating is most often required. Protective functionalities of paperboard can be obtained through the application of coating layers containing binders and pigments. Paper coating formulations are examples of macrocomposites, and such coatings applied on paperboard have been investigated with Peak Force Tapping with the aim of visualizing the distribution of the different coating components at the surface and to elucidate how the drying temperature affects the nature of the surface. ${ }^{48}$ The data reported below was obtained with a simplified coating formulation containing starch and styrene butadiene (SB) latex at a weight ratio of $1: 1$. The latex particles had a diameter of $145 \mathrm{~nm}$ and a glass transition temperature of $18{ }^{\circ} \mathrm{C}$. The coatings were dried either at room temperature or at $105{ }^{\circ} \mathrm{C}$ for 5 minutes. Due to the large size of the particles it was not essential to know the exact tip geometry; however, a tip with a nominal radius of $10 \mathrm{~nm}$ was employed.

The topography image of the sample dried at room temperature is shown in Fig. 6, which displays roughly spherical SB latex particles embedded in a matrix of starch. The latex particles appear to extend about $10 \mathrm{~nm}$ away from the surface (panel A-I). In fact, they extend a bit further since the deformation induced by the tip on top of the latex particles is $\mathbf{1 . 5} \mathbf{n m}$ larger than that encountered over the starch area (panel A-II). In addition, it is noticed that the tip-latex adhesion is larger than that between the tip and the starch (panel A-III). This contributes to the slightly larger dissipation observed on top of the SB latex (panel A-IV). The elastic modulus evaluated on top of the latex particles has a magnitude of $2.2 \pm 0.5 \mathrm{GPa}$ (panel A-V). This value is slightly larger than that obtained by indentation measurements $(1.14 \pm$ $0.03 \mathrm{GPa})^{59}$ and by dynamic mechanical analysis, DMA, (0.48 \pm $0.15 \mathrm{GPa}){ }^{59}$ The elastic modulus of starch determined from the Peak Force Tapping measurements is higher, $3.5 \pm 0.7 \mathrm{GPa}$. This value is between that obtained by a tensile test $(1.1-2.2 \mathrm{GPa})^{60}$ and that obtained by ultrasonic measurements ( $5 \pm 0.05 \mathrm{GPa}) .^{61}$ We emphasize that the AFM-based method provides information on the elastic modulus at the surface region (deformation less than $10 \mathrm{~nm}$ ), whereas other methods such as DMA provide values for the bulk material. In general, the surface elastic modulus and the bulk elastic modulus do not need to be the same, ${ }^{18}$ but in this particular case the values appear to be similar.

The sample dried at $105{ }^{\circ} \mathrm{C}$ for 5 minutes displays a smoother surface (Fig. 6) as the latex particles started to deform to form a surface film. This is most clearly seen in the deformation and elastic modulus images shown in Fig. 6. However, this drying condition was not sufficient to create a uniform film, which often is needed in applications.

Macrocomposite coatings are used in many applications, and below we provide another illustration on how AFM-based techniques can provide valuable information related to a coating designed for corrosion protection purposes. ${ }^{62}$ This coating consisted of a polyester acrylate (PEA) matrix in which small conducting polyaniline (PANI) particles were dispersed. The PEA matrix formed a passive corrosion protection layer, whereas the redox properties of the PANI particles provided additional electroactive corrosion protection. In order to achieve efficient protection, the PANI particles need to form a conductive network that spans the entire coating layer.

In this AFM study ${ }^{62}$ Peak Force Tuna, which is Peak Force Tapping with the additional possibility to measure the local current between tip and sample, was utilized. For this purpose carbon steel was coated with a corrosion protection layer of PEA and polyaniline particles doped with phosphoric acid (PANI-PA), as schematically illustrated in Fig. 7. It should be noted that a current will only be measured if the conductive network spans from the underlying metal substrate to the surface of the coating probed by the AFM tip.

In order for the coating to provide efficient corrosion protection it needs to contain a conductive network, but it also needs to be free of defects and in particular the interphase (the interface region) between particles and matrix needs to be strong. ${ }^{63}$ This is achieved when the interactions between the particles and matrix are favorable, which can be controlled by modification of the particle surface character. Some AFM data acquired around a PANI-PA particle located at the surface of the coating is illustrated in Fig. 8. The topography image shows a large PANI-PA particle $(\approx 0.5-1 \mu \mathrm{m})$ that extends from the interface, and also some smaller particles. In separate AFM measurements a pure PEA film was shown to have a surface elastic modulus of just below $3 \mathrm{GPa}$, whereas a film of pure PANI-PA had a surface elastic modulus of about 6-7 GPa. This elastic modulus difference is clearly visible in the modulus image of the PEA/PANI-PA composite reported in Fig. $8 \mathrm{~b}$. It should be noted that even though this coating provides good corrosion protection, ${ }^{64}$ the low elastic modulus observed on the right hand side of the large particle (Fig. 8b, inset) suggests reduced polymer density and thus a weak interphase which could facilitate the penetration of water and corrosive ions during long term exposure. Both the large PANI-PA particle and several of the smaller particles are clearly connected in a conductive network, as observed in the contact current image. Thus, a network structure similar to that illustrated in Fig. 7 was achieved. By comparing the abundance of PANI-PA particles at the interface towards the air and inside the coating, as visualized on the surfaces created by microtoming, it could be concluded that the PANI-PA particles were depleted from the coating-air interface. ${ }^{62}$ This is a consequence of the lower surface energy of the PEA matrix compared to that of the PANI-PA particles. Thus, images of surfaces obtained by microtoming provide a more accurate picture of the structure inside coatings.

\section{Nanocomposites}

Nanocomposites are distinguished from macrocomposites by the size of the incorporated particles, which are in the nm-range in nanocomposites and in the $\mu \mathrm{m}$-range in macrocomposites. 

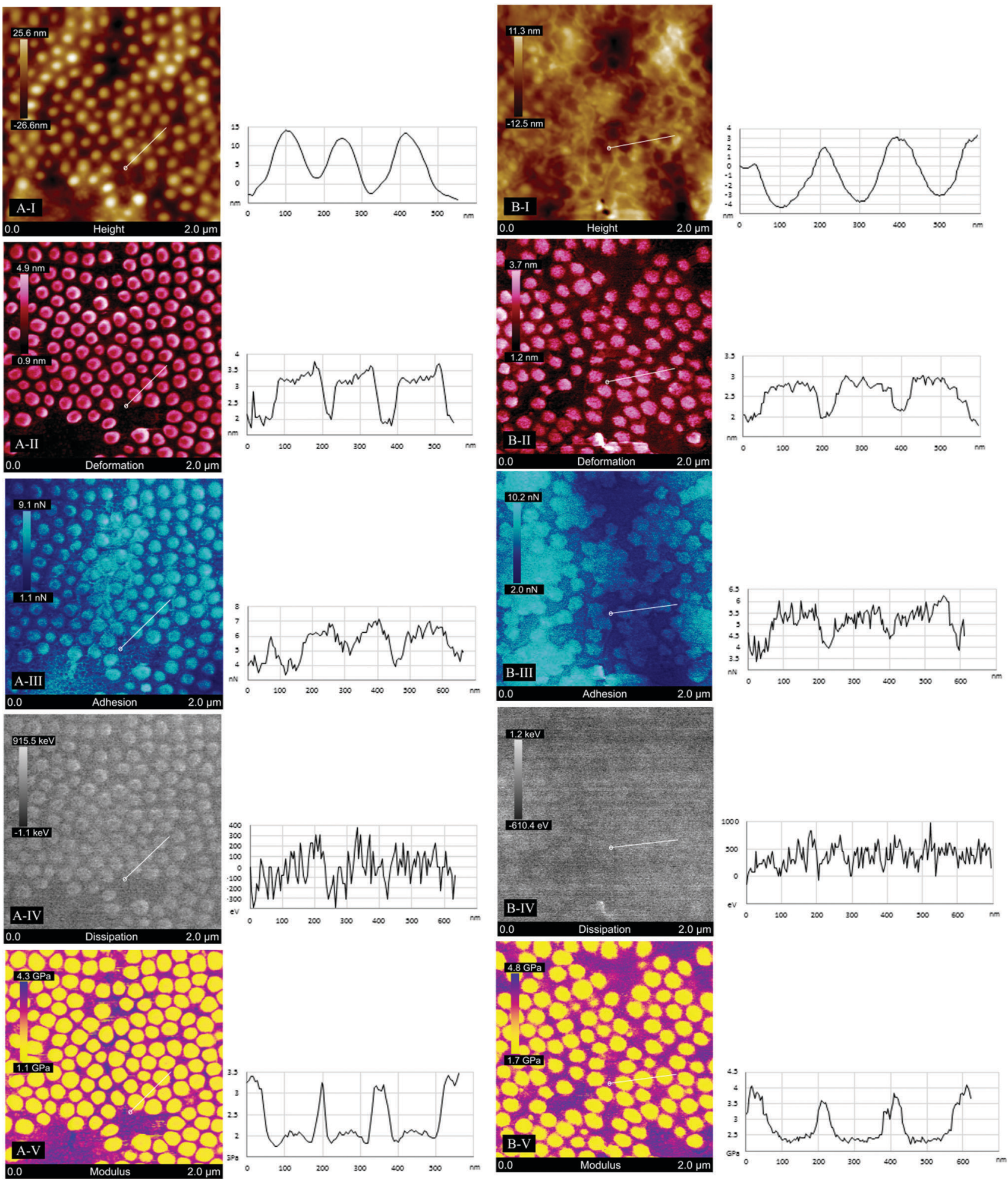

Fig. 6 Peak Force Tapping AFM $2 \times 2 \mu \mathrm{m}^{2}$ images illustrating the effect of drying temperature on mechanical properties. Left column: Starch-latex film dried at room temperature, (A-I) height, (A-II) deformation, (A-III) adhesion, (A-IV) dissipation and (A-V) modulus. Right column: Starch-latex film dried at $105{ }^{\circ} \mathrm{C}$ for 5 minutes, (B-I) height, (B-II) deformation, (B-III) adhesion, (B-IV) dissipation and (B-V) modulus. The line profile to the right of each figure follows the line shown in the corresponding image. Reprinted with permission from ref. 48 Copyright, 2017 American Chemical Society.

This may seem trivial, but the larger surface area for a given volume fraction in nanocomposites results in dramatically different properties. A particular concern when the size of the dispersed particles decreases is the properties of the interphase 


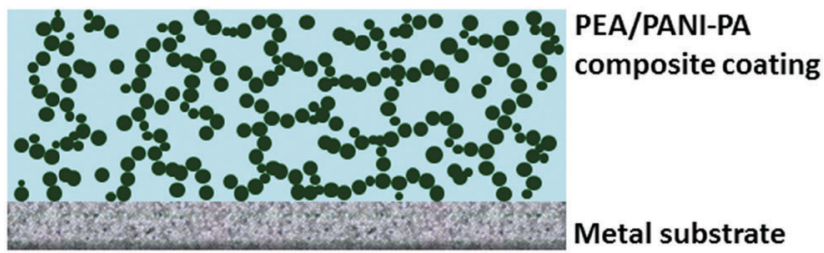

Fig. 7 Schematic of the network of spherical PANI-PA nanoparticles dispersed in a PEA matrix. Reprinted with permission from ref. 62 Copyright, 2017 American Chemical Society.

region, which is due to the rapid increase in total interphase volume when the size of the dispersed particles decreases at a constant volume fraction. Indeed, it has been realized that the interphase properties are key to the performance of nanocomposite materials. ${ }^{63}$ AFM-based techniques, which have the ability to provide local information on material properties, seem to be an excellent choice for such studies and some attempts to evaluate interphase properties are discussed in the next examples.

The quantitative imaging (QI) and force volume mapping modes were applied to elucidate the temperature-dependent surface nanomechanical properties of a nanocomposite consisting of a poly(ethyl methacrylate) (PEMA) and poly(isobutyl methacrylate) (PiBMA) matrix and hydrophobized silica nanoparticle fillers. ${ }^{65}$ The QI images of the nanocomposite surface at different temperatures of $23{ }^{\circ} \mathrm{C}, 40{ }^{\circ} \mathrm{C}$ and $63{ }^{\circ} \mathrm{C}$ during heating and $29{ }^{\circ} \mathrm{C}$ after cooling are shown in Fig. 9, where a few individual particles and a particle cluster are clearly visible. As expected, the stiffness is higher for the silica particles than for the polymer matrix. Similarly, the stiffer particles have lower adhesion to the AFM tip than the softer polymer matrix.

The polymer matrix, as expected, became softer with an increase in temperature, as seen in the measured slope images which report the slope of the repulsive force encountered as the tip indents the surface. In this work it was also attempted to determine the interphase thickness, i.e. the thickness of the polymer matrix region around a particle that has different properties compared to the bulk matrix. To this end, the variation in the stiffness across the polymer/particle surface and interphase area was measured with high lateral resolution (Fig. 10). It can clearly be seen that the stiffness varies smoothly with distance from the particle centre and that the effect extends beyond the particle. It should also be noted that the stiffness measured for the silica particle is much lower than that expected for silica. This is due to the response of the underlying polymer matrix which easily deforms when the particle experiences the compressive force exerted by the tip.

An obstacle that hampers the accurate determination of the interphase thickness arises from the well-known convolution effect between the cantilever tip and the surface feature (Fig. 10a). Thus, a conservative estimate of the interphase thickness is obtained by only considering data that goes beyond point $\mathrm{B}$ in the topography image, which corresponds to point $\mathrm{C}$ in the stiffness image (Fig. 10b). The interphase thickness is then estimated as the distance between point B and point C (Fig. 10b). This method was utilized to estimate the variation of the interphase thickness with temperature, as shown in Fig. 10c. It was found that the interphase thickness increased with temperature, possibly due to the increase in the difference in the stiffness measured over the particle and over the polymer matrix which results in increased stiffness contrast. Similar data were achieved with both QI and force volume mapping AFM, and the differences noted between these two modes were attributed to the slower probing speed in the force volume mapping mode which allowed larger surface deformation to occur during measurements.

In the next example we illustrate how a multifrequency scanning probe method, ImAFM, can be used to map surface mechanical variations across a nanocomposite surface. In this example hydrophobic $16 \mathrm{~nm}$ silica particles were incorporated in a soft PDMS matrix and no contact mechanics model was applied in the data analysis. Such an approach can be advantageous in the (a)

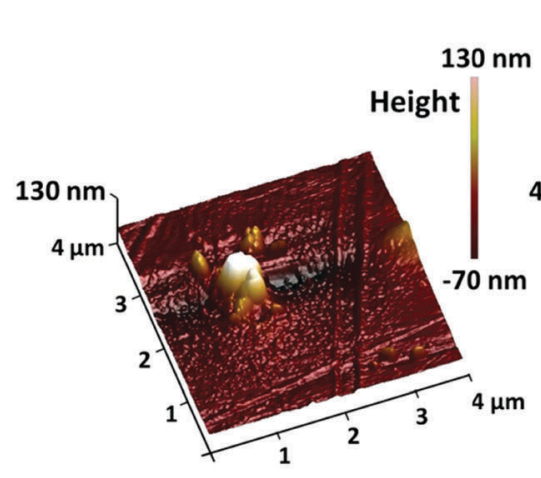

(b)

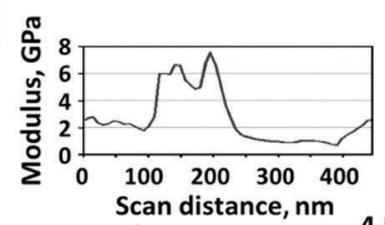

(c)
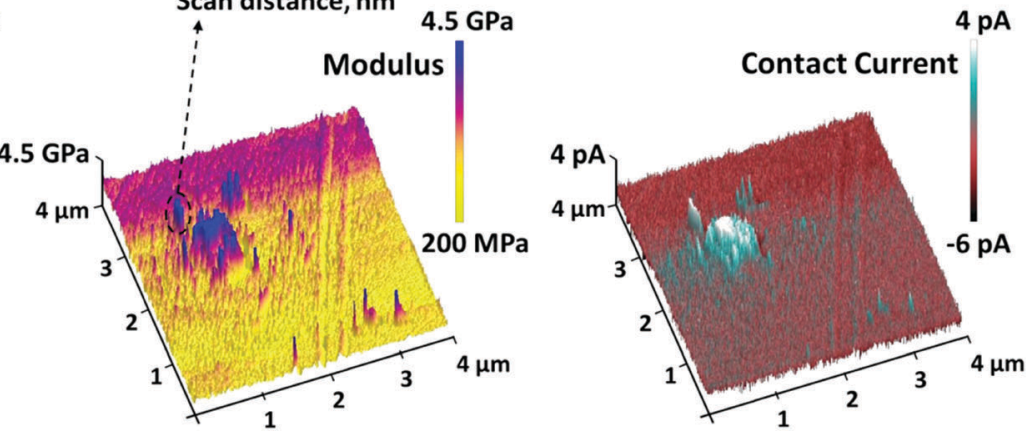

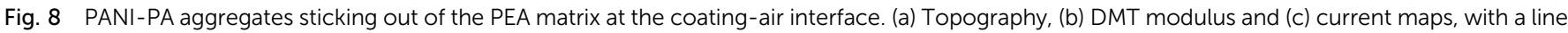

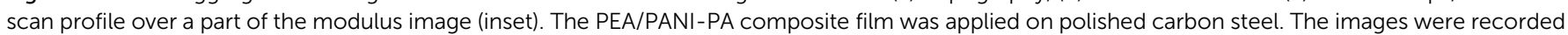
in air using Peak Force Tapping TUNA. Reprinted with permission from ref. 62 Copyright, 2017 American Chemical Society. 

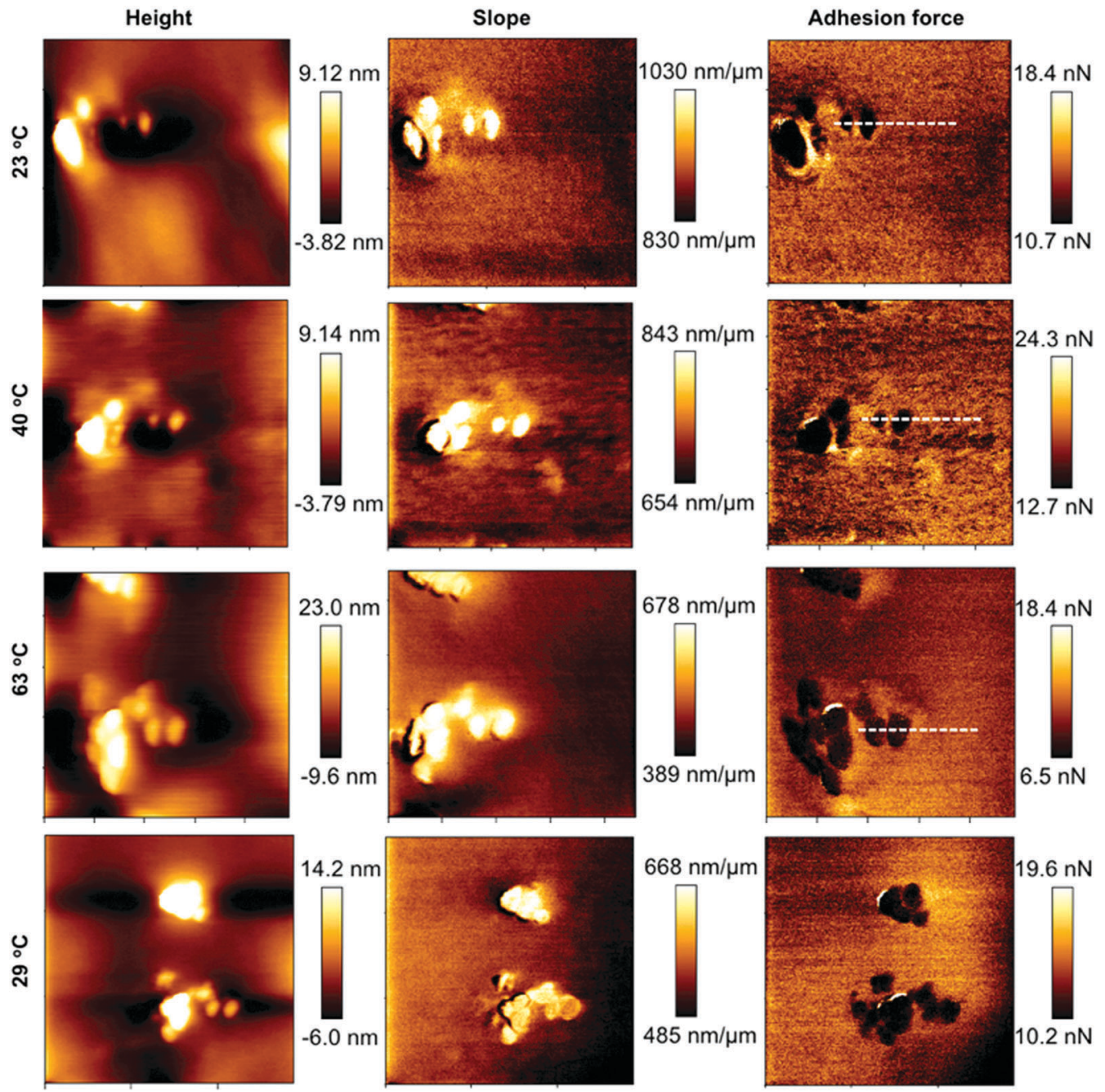

Fig. 9 Q images of the nanocomposite layer showing height (left column), slope/stiffness (middle column) and adhesion force (right column). The scan size is around $500 \mathrm{~nm} \times 500 \mathrm{~nm}$ for all images. From top to bottom the different rows correspond to images recorded at $23{ }^{\circ} \mathrm{C}$, $40{ }^{\circ} \mathrm{C}$ and $63{ }^{\circ} \mathrm{C}$ and $29^{\circ} \mathrm{C}$ after cooling the sample again. The scan areas are not exactly the same due to some drift, but the particles marked by the dashed lines are observed in all images. Reprinted with permission from ref. 65 (C) 2017 Elsevier Inc.

case of soft matter materials, where the surface may respond to the approaching tip prior to contact by deforming towards the tip due to attractive van der Waals or capillary forces, ${ }^{66}$ which are not considered in classical contact mechanics models.

In this example we illustrate the contrast achieved in one model independent surface nanomechanical property, namely the $F_{\mathrm{I}}$-stiffness, which is defined as the slope of the $F_{\mathrm{I}}$ curve at high amplitudes, as seen in Fig. 2. Two $F_{\mathrm{I}}$-stiffness maps for the pure PDMS surface and for the nanocomposite with added nanoparticles are shown in Fig. 11(a and b). The $F_{\mathrm{I}}$-stiffness map for the pure PDMS samples demonstrates that the stiffness property is around 4 to $12 \mathrm{mN} \mathrm{m}^{-1}$ and a clear contrast between the two distinct areas with lower and slightly higher stiffness can be observed. The $F_{\mathrm{I}}$-stiffness analysis on the nanocomposite with silica particles embedded in the PDMS matrix shows that the polymer matrix became a bit stiffer in comparison to the pure PDMS sample. Moreover, a clear contrast between the matrix and the silica particles is achieved in the map shown in Fig. 11(b). Small silica particles of much higher $F_{\mathrm{I}}$-stiffness are clearly visible, but some areas with higher stiffness in between the matrix and silica particles can also clearly be observed. The direct measurement of the $F_{\mathrm{I}}$-stiffness is a simple route to visualize surface property variations over the nanocomposite surface.

\section{Hydrogels}

Hydrogels are very soft and challenging to investigate with AFMbased techniques since classical contact mechanics models are not applicable. In this case, the newly proposed model that considers the motion of the surface due to the influence of long-range forces may be appropriate. ${ }^{66}$ In this section we do not present any detailed nanomechanical analysis of hydrogel materials, but point out some features of hydrogel surfaces that require particular attention in forthcoming work.

The ImAFM method was employed to study a (polyhydroxyethyl methacrylate) (poly-HEMA) hydrogel surface at 18\% humidity, as 
(a)
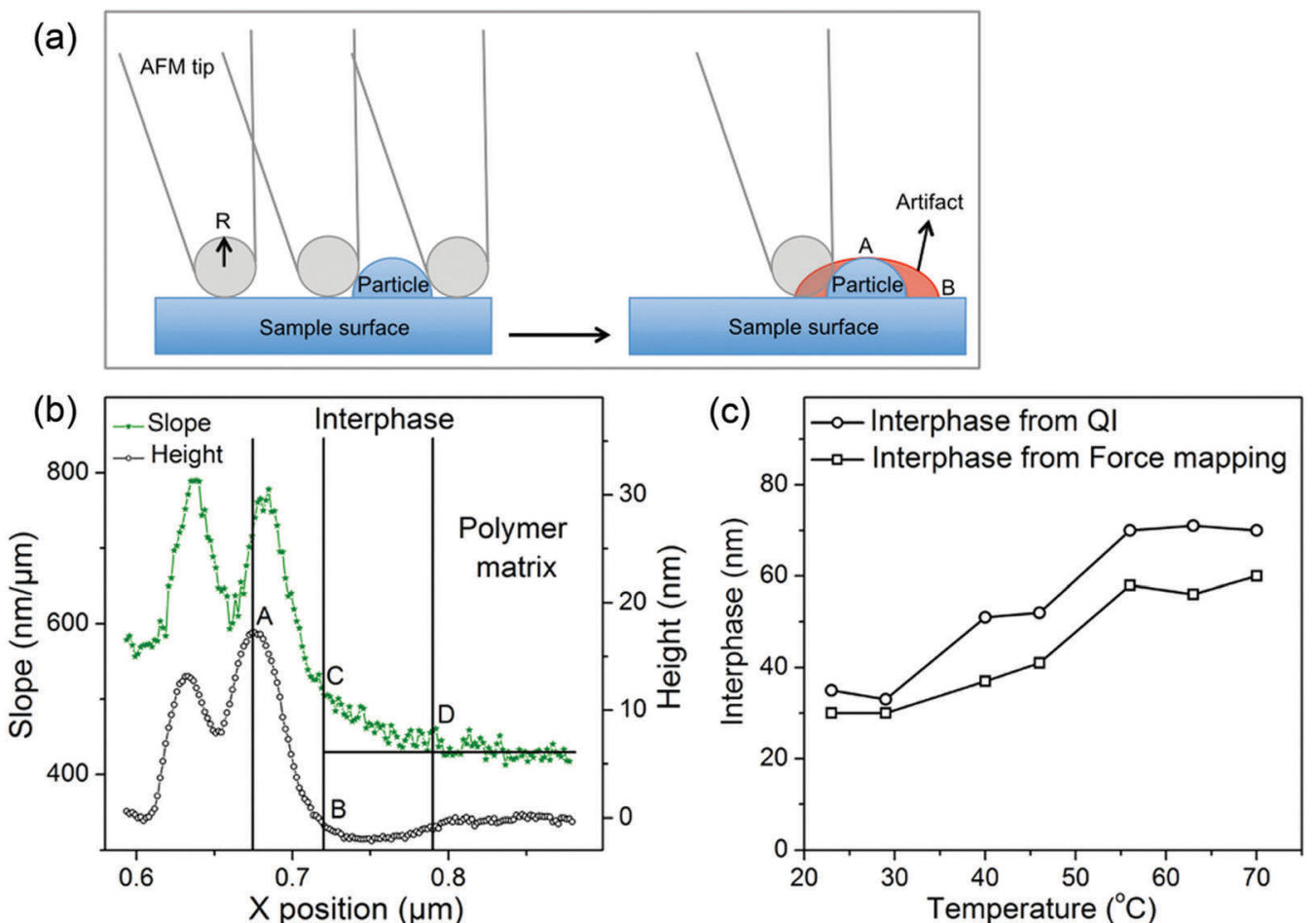

Fig. 10 (a) Schematic showing an AFM artefact when using a tip with similar dimensions (tip radius, $R \approx 15 \mathrm{~nm}$ ) to the particle diameter (40 nm). (b) Illustration showing how the interphase region is determined from the data presented in Fig. 6A and $\mathrm{B}$. The horizontal line is defined by the mean value of the measured slope/stiffness on the polymer matrix. The height profile is taken as a reference to define the edge of the particle. Points A and B in (a) correspond to the same points in the height profile. The distance between points $C$ and $D$ in the stiffness profile is defined as the interphase thickness. (c) Temperature dependence of the thickness of the interphase region determined from QI and force volume mapping mode. Reprinted with permission from ref. 65 (C) 2017 Elsevier Inc.

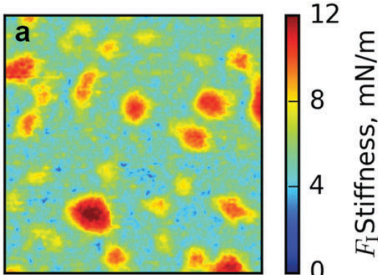

$1 \mu \mathrm{m} \times 1 \mu \mathrm{m}$

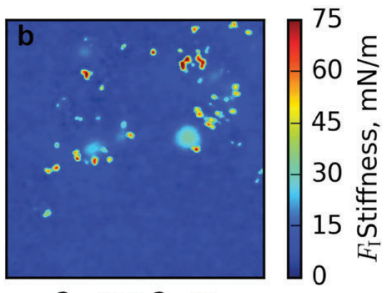

$2 \mu \mathrm{m} \times 2 \mu \mathrm{m}$
Fig. 11 (a and b) Maps of $F_{1}$-stiffness distribution over pure PDMS surface (a) and over the PDMS hydrophobic silica nanocomposite surface (b). Note the different stiffness scales in the two images.

measured in the sample chamber and the humidity environment was controlled with a humidifier (Cellkraft, Sweden). The polyHEMA hydrogel was synthesized using HEMA monomer (60.7\%), azobisisobutyronitrile (initiator, $0.3 \mathrm{wt} \%$ ), poly(ethylene glycol) diacrylate (cross-linking agent, $3 \mathrm{wt} \%$ ) and water (36 wt\%). A thin film of the mixed solution was cured between two glass slides in an oven at $65{ }^{\circ} \mathrm{C}$ for 4 hours to form the hydrogel material. The same surface area of the hydrogel was scanned twice and the corresponding phase images are shown in Fig. 12(a-d).

The first inherent complication observed for the hydrogel sample is that the surface is already noticeably mobile at $18 \%$ humidity, and as a consequence the surface features do not appear the same on the first and second scan. This is also observed at higher humidity. The phase contrast as measured with ImAFM clearly demonstrates two distinct areas in both images, with higher and lower negative phases, which can be interpreted as softer, the blue areas, and stiffer, the red areas. The corresponding $F_{\mathrm{I}}(A)$ and $F_{\mathrm{Q}}(A)$ force quadratures taken on these areas are shown in Fig. 12(b-f). The force quadrature curves shown with solid lines were taken at the points marked with red and blue crosses during the first scan, whereas the force quadrature curves shown with dashes were taken on the points marked with red and blue filled circles during the second scan of the same area. Although a clear net repulsive behavior is observed for the stiffer areas at higher amplitudes on the $F_{\mathrm{I}}(A)$ curve, almost no net repulsion or weak repulsion is observed over the softer blue areas at the highest amplitude applied in this experiment. On the softer areas there is also a clear hysteresis observed in the $F_{\mathrm{I}}(A)$ curve between measurements done while increasing and decreasing the amplitude. This suggests that when the tip touches the surface of the soft areas, it moves together with the surface and the softer surface areas are not fully relaxed after such interactions. Thus, the surface position changes due to the tip-surface interaction during the measurement, which gives rise to the hysteretic behavior. However, the $F_{\mathrm{I}}(A)$ curves on the softer areas are similar for the first and second scans. Similar trends are observed in the $F_{\mathrm{Q}}(A)$ curves, where no hysteresis in the dissipation energy is observed for the stiffer areas, whereas a significant hysteresis is observed on the soft areas. 

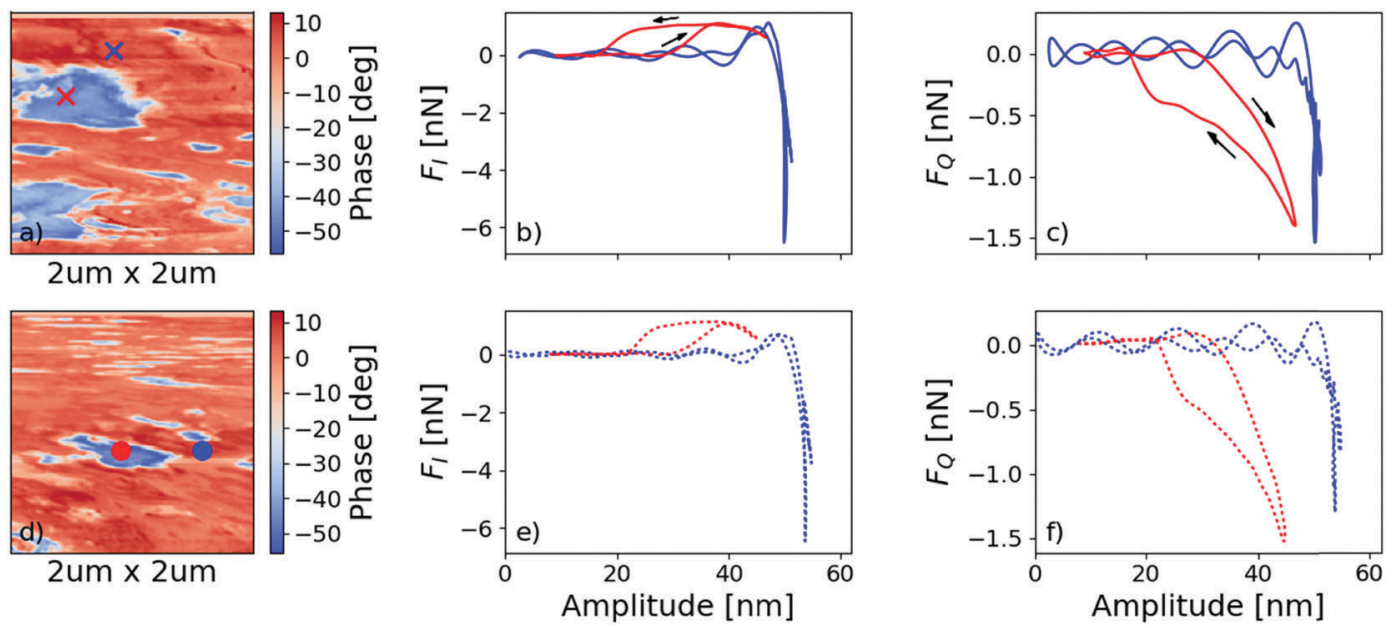

Fig. 12 (a-f) Two distinct regions, blue for higher negative phase and red for lower negative phase, are observed in the phase images measured on the same poly-HEMA hydrogel surface area during the first scan (a) and the second scan (d). $F_{1}(A)\left(b\right.$ and e) and $F_{Q}(A)$ (c and f) force quadratures recorded at the pixel in the phase maps marked with the blue and red crosses for the first scan and filled circles for the second scan. The colors of the force quadratures correspond to the colors of the markers. The two black arrows shown in (b and c) demonstrate when the $F_{1}(A)$ and $F_{Q}(A)$ curves were collected during amplitude increase and decrease, respectively.

A plausible interpretation of the data shown in Fig. 12 is that the different areas represent surface regions with different amounts of water, and the softer regions correspond to areas with a higher surface water content. Recently, Haviland $e t$ al. introduced a moving surface model to analyze measured $F_{\mathrm{I}}(A)$ and $F_{\mathrm{Q}}(A)$ force quadratures to extract local nanomechanical properties on such soft materials, ${ }^{66}$ and future works on hydrogels could benefit from comparison with predictions of such models.

\section{Local wear}

Reducing wear is of paramount importance for any material, particularly for surfaces that slide against each other, and wear is a common cause of failure for protective coatings. AFM-based investigations provide a new avenue for investigating local wear and for providing information related to the initiation of wear. This may be particularly important for composite systems where wear may be initiated in the matrix phase, by removing dispersed particles located at the sliding surfaces or perhaps in the interphase region. AFM-based studies have the potential to address this important topic, and this area of research is gaining increasing interest. ${ }^{67-70}$

The first example that we will discuss is concerned with a thin $(<100 \mathrm{~nm})$ composite layer where a mussel adhesive protein was utilized for binding ceria nanoparticles to carbon steel surfaces and to each other. ${ }^{71}$ The interest in such layers arises from the finding that they provide good corrosion protection, as discussed in several recent articles. ${ }^{72-74}$ The basic principle of this type of wear study is to first make a topographic image of the surface over a relatively large surface area $\left(10 \times 10 \mu \mathrm{m}^{2}\right.$ in Fig. 13). The tip is then scanned across a smaller area $\left(1 \times 1 \mu \mathrm{m}^{2}\right.$ in Fig. 13) of the surface in contact mode under a controlled force. Subsequently, the tip is lifted from the surface and moved to another area where it is scanned across the surface at a higher force. This process is then repeated using successively higher forces. Finally, the large surface is imaged again, and the wear scars are analysed.

For the particular case of the nanocomposite film formed by mussel adhesive proteins and ceria nanoparticles shown in Fig. 13, it was observed that at low pressures (force divided by the cross-section area of the tip) the film was already deformed plastically, which resulted in a smoother surface. Abrasion was initiated at a pressure of about $80 \mathrm{MPa}$, and the roughness of the remaining layer increased at higher pressures as wear particles were found on the scanned area. At the highest pressure applied, the average wear scar was about $10 \mathrm{~nm}$ deep, and a significant amount of wear particles could be found at the edge of the scanned area.

There are a few issues related to these types of measurements. First, the conversion of the applied force into pressure is not trivial. Even if the tip shape is accurately known it is not clear how much of the tip is in contact with the sample, and the pressure is not uniform over the entire tip-surface contact area. It may thus be less ambiguous to report the applied force rather than the pressure, but this makes it more difficult to compare measurements performed with different tips. Another issue is that not only the surface but also the tip may experience wear during the measurements. Thus, it is advisable to determine the tip shape both before and after the measurements, which is particularly important when hard samples are investigated.

The next example concerns the local wear of a polymer coating at the nanoscale. This topic was recently reviewed by D'Acunto $\mathrm{et} \mathrm{al}^{75}$ The wear measurement was conducted using a normal force of $0.66 \mu \mathrm{N}$ and $1.32 \mu \mathrm{N}$ on a high molecular weight acrylic polyester with a melamine cross linker, which was supplied by PTE Coatings AB (Västervik, Sweden) and cured at $180{ }^{\circ} \mathrm{C}$ for $10 \mathrm{~min}$ and $30 \mathrm{~min}$ (see Fig. 14). The topography and elastic modulus of this coating is reported in Fig. 5. The wear experiment was conducted in contact mode using only 

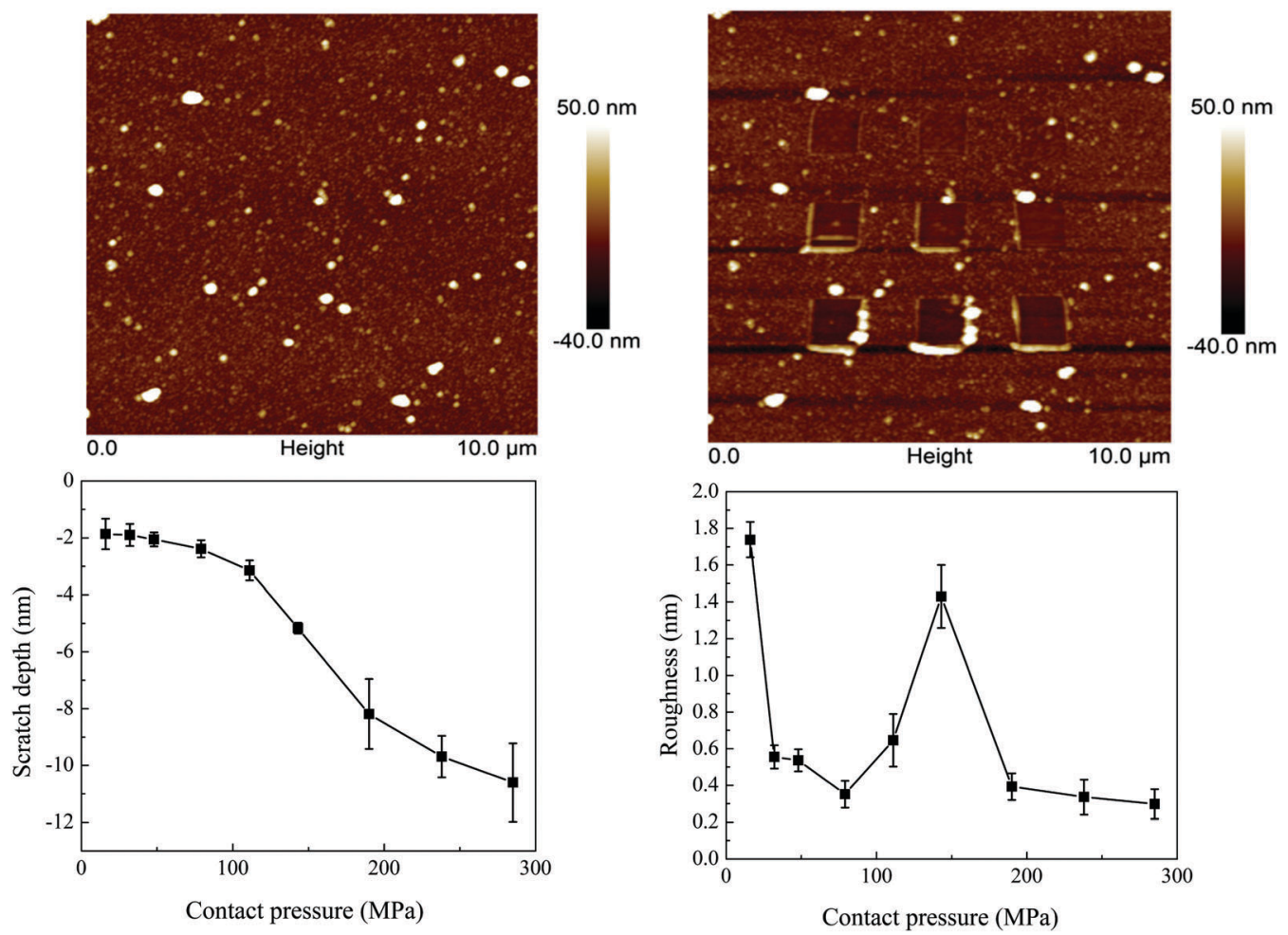

Fig. 13 Surface topography before (top left) and after (top right) wear measurements with different applied contact pressures, which are marked (in MPa) next to the different areas. Bottom left: Average wear depth vs. contact pressure and (bottom right) roughness in the worn area vs. contact pressure. The error bars are based on three separate measurements. Reprinted with permission from ref. 71 Copyright, 2017 American Chemical Society.
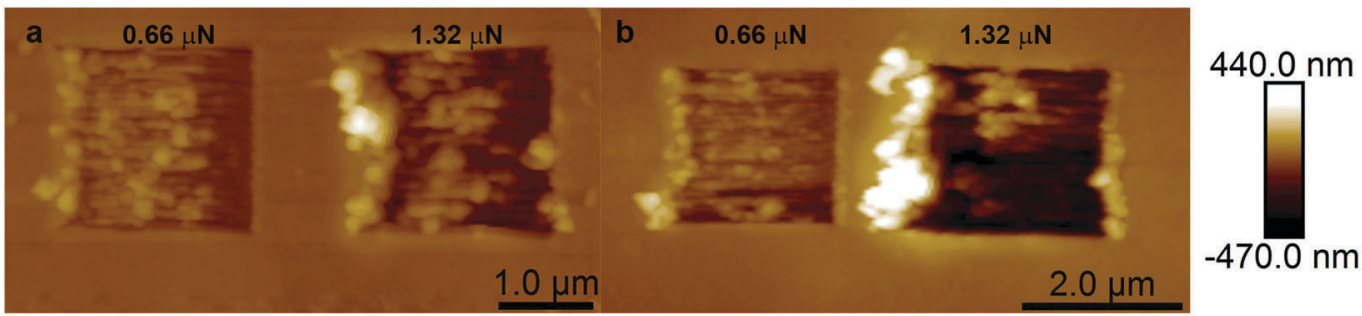

Fig. 14 Surface topography images after the wear test acquired with AFM for the sampled cured for 30 min (a) and for the sample cured for 10 min (b).

one full scan in the slow scan direction at a speed of $2 \mathrm{~Hz}$. We find a correlation between the higher modulus for the sample cured for $30 \mathrm{~min}$ compared to that cured for $10 \mathrm{~min}$ and a smaller wear scar depth for the former sample. The maximum wear depth for the sample cured for $10 \mathrm{~min}$ was $240 \mathrm{~nm}$ at a normal load of $0.66 \mu \mathrm{N}$ and $490 \mathrm{~nm}$ at $1.32 \mu \mathrm{N}$, whereas the corresponding values for the sample cured for 30 min were $110 \mathrm{~nm}$ and $210 \mathrm{~nm}$, respectively. The destruction and removal of material is not homogenous and many wear particles are observed.

\section{Model-dependent or model- independent data evaluation}

Nanomechanical characterization of samples with AFM is based on the analysis of force curves, and data directly obtained from such curves include quantities such as adhesion force, work of adhesion, indentation depth and stiffness calculated as the slope of the force in the contact region. This has the advantage that material properties are evaluated without the use of any contact mechanics model. However, if more generally known quantities are required, such as the elastic modulus of the surface region, a contacts mechanics model has to be applied and this model fitted to the measured force curve. The accuracy of the parameters extracted from the model fit is strongly dependent on the validity of the particular model to describe the tip-surface interaction. In particular, models such as DMT and JKR assume that the tip-surface interaction is purely elastic, whereas viscous and plastic effects play a role for many real systems. Such soft and viscous systems require more complicated tip-surface interaction models, as recently discussed by Haviland et $a .^{66}$ In this context it is also an advantage to separate in-phase and out-of-phase interactions, which is possible with intermodulation AFM. 


\section{Cross-talk}

Another known difficulty with scanning probe based measurements is the interdependence of different measured quantities, among which the most well-known is the interdependence between deformation and topography. This arises from the fact that topography is measured while keeping a control parameter constant, e.g. constant force in contact mode and Peak Force Tapping and constant amplitude reduction in tapping mode and constant working distance in ImAFM. Since materials with different elastic moduli will deform differently due to the interactions with the tip, this will lead to an error in the topography image where the softer material will deform more and thus appear to be at a lower height in the topography image. When the stiffness contrast is large this can, if not corrected for, lead to misleading results. For instance, in our unpublished studies of hard silica nanoparticles in a soft PDMS matrix the topography image suggested that the particles were sticking out from the polymer matrix. However, this was solely an artefact due to the softer nature of the matrix, and when corrected for it was instead noticed that the particles were imbedded in the matrix.

As mentioned previously in this article, and as also can be seen from Fig. 1, a strong adhesive force will result in hysteresis between the forces measured on approach and on separation. This hysteresis is reported as the energy dissipation during one force cycle, thus it is clear that an increase in adhesion force also will make a contribution to increased energy dissipation. This is even clearer when considering the work of adhesion, which is defined as the area below the interpolated free-force level on the retraction force curve, whereas the dissipation energy is the area between approach and retraction force curves.

In quasi-static force measurements using large surfaces, it is wellknown that when the gradient of the force exceeds the spring constant, the mechanical system becomes unstable and there is a jump in the force curve that does not reflect the actual surface forces. ${ }^{76}$ This problem is less severe when sharp tips are used. For instance, if we consider a van der Waals interaction characterized by a Hamaker constant of $1 \times 10^{-19}(\mathrm{~J})$ between a tip with a radius of $20 \mathrm{~nm}$ mounted on a cantilever with a spring constant of $2 \mathrm{~N} \mathrm{~m}^{-1}$, a spring instability resulting in a jump-in would occur at a distance of about $0.7 \mathrm{~nm}$ and on separation the jump-out distance would be $5 \mathrm{~nm}$ if the adhesion force is $10 \mathrm{nN}$. This situation is illustrated in Fig. 15, where the true force curve is shown as a solid line and the jump-in and jump-out trajectories are shown by dashed lines. Thus, in this case there would be an artificial hysteresis between the approach and retract force curves, which would be interpreted as energy dissipation. However, this hysteresis has nothing to do with surface properties but results solely from the spring instability. This effect becomes more important with a lower spring constant, larger tip radius and stronger adhesion force.

\section{Determination of tip geometry}

In order to carry out quantitative nanomechanical characterization, it is of importance to know the tip geometry accurately since this has a significant impact on the properties evaluated in both

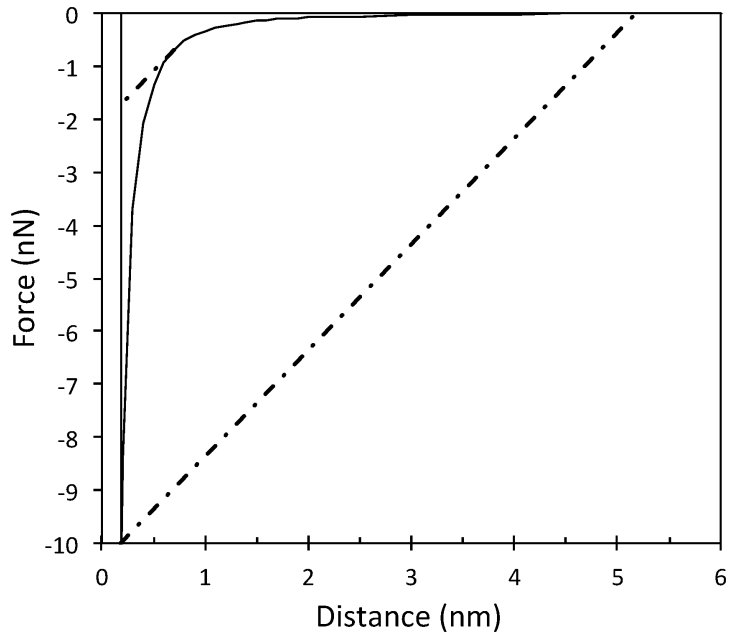

Fig. 15 The van der Waals force between a tip and flat surface as a function of separation (solid line). Mechanical instability occurs on approach and the tip follows the path shown as the upper dashed line. Another instability occurs on separation where the tip follows the lower dashed line. Thus, the complete force curve cannot be traced accurately and a hysteresis due to spring instabilities occurs.

model-independent and model-dependent approaches. ${ }^{77}$ The specific tip geometry consequently has to be given as an input parameter when fitting contact mechanics models to measurements. There are two principally different ways to take into account the tip shape. The direct way is to measure the tip shape using high resolution SEM or to reconstruct the tip shape using a surface with very sharp features, and some AFM manufacturers can provide such surfaces. However, the use of tip-sample convolution to determine the tip shape also requires careful experimental considerations. ${ }^{78,79}$ An indirect method is to use a sample with a known elastic modulus and then adjust the tip radius until the contact mechanics model chosen for analysis returns an elastic modulus that is in agreement with the known value. The same tip can then be used for characterizing an unknown sample. If this approach is used, the calibration sample and the unknown sample should have similar elastic moduli, thus given experimental parameters lead to similar indentation depths. A drawback with this method is that it assumes that the surface elastic modulus is the same as the bulk elastic modulus, which not necessarily is correct. ${ }^{18}$

During AFM measurements, particularly on stiff surfaces, the tip can become worn due to high friction and compression. This will change the tip shape and as a result cause an error in data evaluation based on the initial tip geometry. Thus, the quality of the tip should be checked also after measurements. This can be done as described above and also by performing measurements on a standard sample before and after performing the measurement on the sample of interest. If no wear has occurred the same property maps with the same resolution should be obtained on the standard sample in both cases.

\section{Conclusions}

In this perspective article we have described how scanning probe methods can be utilized for probing the local material 
properties of a vast range of surfaces, ranging from very soft hydrogel surfaces to stiff samples. Such measurements are of importance in many areas, and our examples have covered packaging materials, corrosion protective coatings and studies of local wear. The elucidation of the property variations over a surface is of particular relevance for composite layers, where surface nanomechanical properties vary significantly and AFMbased techniques can readily visualize this. There are several different AFM modes that can be used for such measurements, which have advantages and disadvantages. For quantitative measurements, care has to be taken to determine the tip shape, preferably both before and after the measurements. In many cases contact mechanics models are utilized to extract wellknown properties such as the Young's modulus. However, it should be kept in mind that these models involve several assumptions, including an elastic response of the surface in most classical models. In reality this is often not the case, and under such circumstances the intermodulation method has the advantage that in-phase and quadrature forces can be separated, but with this comes the cost of not recording intuitively simple force $v s$. distance curves but rather less intuitive time-average force vs. amplitude curves. Currently large efforts are being placed into developing both measurement techniques and contact mechanics models, and we foresee that significant technical, experimental and theoretical advances will be made within the coming years, which will impact both fundamental and applied science.

\section{Acknowledgements}

PC acknowledges financial support from the Swedish Research Council (VR). DBH acknowledges support from the Olle Enqvist Foundation. Both PMC and DBH acknowledge support and equipment donated by the Knut and Alice Wallenberg Foundation. PTE Coatings AB (Västervik, Sweden) is thanked for gifting polymer coatings.

\section{References}

1 J. Gao, W. D. Luedtke, D. Gourdon, M. Ruths, J. N. Israelachvili and U. Landman, J. Phys. Chem. B, 2004, 108, 3410-3425.

2 J. N. Israelachvili, Intermolecular and Surface Forces, Elsevier, Amsterdam, 3rd edn, 2011.

3 B. V. Derjaguin and L. Landau, Acta Physicochim. URSS, 1941, 14, 633-662.

4 E. J. N. Verwey and J. T. G. Overbeek, Theory of Stability of Lyophobic Colloids, Elsevier, Amsterdam, 1948.

5 I. E. Dzyaloshinskii, E. M. Lifshitz and L. P. Pitaevskii, Adv. Phys., 1961, 10, 165-209.

6 V. A. Parsegian, Van der Waals Forces. A Handbook For Biologists, Chemists, Engineers, And Physicists, Cambridge University Press, Cambridge, 2006.

7 P.-G. de Gennes, Scaling concepts in polymer physics, Cornell University Press, Ithaca, 1979.

8 G. J. Fleer, M. A. Cohen Stuart, J. M. H. M. Scheutjens, T. Cosgrove and B. Vincent, Polymers at Interfaces, Chapman \& Hall, London, 1993.
9 J. N. Israelachvili and G. E. Adams, J. Chem. Soc., Faraday Trans. 1, 1978, 74, 975-1001.

10 W. A. Ducker, T. J. Senden and R. M. Pashley, Nature, 1991, 353, 239-241.

11 G. Binnig, C. F. Quate and C. Gerber, Phys. Rev. Lett., 1986, 56, 930-933.

12 P. K. Hansma, J. P. Cleveland, M. Radmacher, D. A. Walters, P. E. Hillner, M. Bezanilla, M. Fritz, D. Vie and H. G. Hansma, Appl. Phys. Lett., 1994, 64, 1738-1740.

13 J. Tamayo and R. Garcia, Langmuir, 1996, 12, 4430-4435.

14 H. Hertz, J. Reine Angew. Math., 1881, 92, 156-171.

15 B. V. Derjaguin, V. M. Muller and Yu. P. Toporov, J. Colloid Interface Sci., 1975, 53, 314-326.

16 K. L. Johnson, K. Kendall and A. D. Roberts, Proc. R. Soc. London, Ser. A, 1971, 324, 301-313.

17 I. N. Sneddon, Proc. Cambridge Philos. Soc., 1948, 44, 492-507.

18 D. Hoffman, I. Miskioglu, K. E. Aifantis and J. Drelich, J. Adhes. Sci. Technol., 2012, 26, 1201-1220.

19 J. Brinell, Gießlers Baumaterilienkunde, 1900, 5.

20 A. Shore and R. Hadfield, J. Iron Steel Inst., London, 1918, 59.

21 R. L. Smith and G. E. Sandly, Proceedings of the Institution of Mechanical Engineers, 1922, 102, 623-641.

22 F. Knoop, C. G. Peters and W. B. Emerson, J. Res. Natl. Bur. Stand., 1939, 23, 39-61.

23 S. M. Walley, Mater. Sci. Technol., 2012, 28, 1028-1044.

24 I. Manika and J. Maniks, Acta Mater., 2006, 54, 2049-2056.

25 G. M. Pharr and F. R. Brotzen, J. Mater. Res., 1992, 7, 613-617.

26 G. M. Pharr and W. C. Oliver, MRS Bull., 1992, 17, 28-33.

27 G. M. Pharr, Mater. Sci. Eng., A, 1998, 253, 151-159.

28 W. C. Oliver and G. M. Pharr, J. Mater. Res., 2004, 19, 3-20. 29 J. Menčík, D. Munz, E. Quandt, E. R. Weppelmann and M. V. Swain, J. Mater. Res., 1997, 12, 2475-2484.

30 M. R. Vanlandingham, S. H. McKnight, G. R. Palmese, J. R. Elings, X. Huang, T. A. Bogetti, R. F. Eduljee and J. W. Gillespie Jr, J. Adhes., 1997, 64, 31-59.

31 N. A. Burnham and R. J. Colton, J. Vac. Sci. Technol., A, 1989, 7, 2906-2913.

32 B. Bhushan and V. N. Koinkar, Appl. Phys. Lett., 1994, 64, 1653-1655.

33 N. Almqvist, Y. Delamo, B. L. Smith, N. H. Thomson, A. Bartholdson, R. Lal, M. Brzezinski and P. K. Hansma, J. Microsc., 2001, 202, 518-532.

34 M. Chyasnavichyus, S. L. Young and V. V. Tsukruk, Jpn. J. Appl. Phys., 2015, 54, 08LA02.

35 M. E. McConney, S. Singamaneni and V. V. Tsukruk, Polym. Rev., 2010, 50, 235-286.

36 D. Maugis and B. Gauthier-Manuel, J. Adhes. Sci. Technol., 1994, 8, 1311-1322.

37 I. N. Sneddon, Int. J. Eng. Sci., 1965, 3, 47-57.

38 R. Garcia and R. Perez, Surf. Sci. Rep., 2002, 47, 197-301.

39 J. Tamayo and R. Garcia, Langmuir, 1996, 12, 4430-4435.

40 G. Bar, Y. Thomann, R. Brandsch, H.-J. Cantow and M.-H. Whangbo, Langmuir, 1997, 13, 3807-3812.

41 A. San Paulo and R. García, Phys. Rev. B: Condens. Matter Mater. Phys., 2001, 64, 193411. 
42 A. F. Payam, D. Martin-Jimenez and R. Garcia, Nanotechnology, 2015, 26, 185706.

43 M. E. Dokukin and I. Sokolov, Langmuir, 2012, 28, 16060-16071.

44 T. J. Young, M. A. Monclus, T. L. Burnett, W. R. Broughton, S. L. Ogin and P. A. Smith, Meas. Sci. Technol., 2011, 22, 125703.

45 L. Chopinet, C. Formosa, M. P. Rols, R. E. Duval and E. Dague, Micron, 2013, 48, 26-33.

46 M. Radmacher, J. P. Cleveland, M. Fritz, H. G. Hansma and P. K. Hansma, Biophys. J., 1994, 66, 2159-2165.

47 K. Nakajima, M. Ito, D. Wang, H. Liu, H. K. Nguyen, X. Liang, A. Kumagai and S. Fujinami, Microscopy, 2014, 63, 193-207.

48 M. Sababi, J. Kettle, H. Rautkoski, P. M. Claesson and E. Thormann, ACS Appl. Mater. Interfaces, 2012, 4, 5534-5541.

49 N. F. Martinez, S. Patil, J. R. Lozano and R. Garcia, Appl. Phys. Lett., 2006, 89, 153115.

50 R. Proksch, Appl. Phys. Lett., 2006, 89, 113121.

51 D. Forchheimer, R. Forchheimer and D. B. Haviland, Nat. Commun., 2015, 6, 6270.

52 D. Ebeling, B. Eslami and S. De Jesus Solares, ACS Nano, 2013, 11, 10387-10396.

53 D. Platz, D. Forchheimer, E. A. Tholén and D. B. Haviland, Nat. Commun., 2013, 4, 1320.

54 D. Platz, D. Forchheimer, E. A. Tholén and D. B. Haviland, Nat. Commun., 2013, 4, 1360.

55 D. Forchheimer, R. Forchheimer and D. B. Haviland, Nat. Commun., 2015, 6, 6270.

56 D. Platz, D. Forchheimer, E. A. Tholén and D. B. Haviland, Nanotechnology, 2012, 23, 265705.

57 S. S. Borysov, D. Platz, A. S. de Wijn, D. Forchheimer, E. A. Tolén, A. V. Balatsky and D. B. Haviland, Phys. Rev. B: Condens. Matter Mater. Phys., 2013, 88, 115405.

58 G. Dunér, E. Thormann, A. Dedinaite, P. M. Claesson, K. Matyjaszewski and R. D. Tilton, Soft Matter, 2012, 8, 8312-8320.

59 A. M. Forster, S. Watson and J. White, in Nanotechnology Applications in coatings, ed. R. H. Fernando and L.-P. Sung, American Chemical Society, Washington, DC, 2009, vol. 1008, pp. 274-290.

60 C.-H. Chen, W.-S. Kuo and L.-S. Lai, Food Hydrocolloids, 2010, 24, 200-207.
61 B. Chen and J. R. G. Evans, Carbohydr. Polym., 2005, 61, 455-463.

62 S. Jafarzadeh, P. M. Claesson, P.-E. Sundell, J. Pan and E. Thormann, ACS Appl. Mater. Interfaces, 2014, 6, 19168-19175.

63 H. Liu and L. C. Brinson, Compos. Sci. Technol., 2008, 68, 1502-1512.

64 S. Jafarzadeh, P. M. Claesson, P.-E. Sundell, E. Tyrode and J. Pan, Prog. Org. Coat., 2016, 90, 154-162.

65 H. Huang, I. Dobryden, N. Ihmer, M. Johansson, H. Ma, J. Pan and P. M. Claesson, J. Colloid Interface Sci., 2017, 494, 204-214.

66 D. B. Haviland, C. A. van Eysden, D. Forchheimer, D. Platz, H. G. Kassa and P. Leclére, Soft Matter, 2016, 12, 619-624.

67 J. L. Loubet, M. Belin, R. Durand and H. Pascal, Thin Solid Films, 1994, 253, 194-198.

68 B. Bhushan, Int. Mater. Rev., 1999, 44, 105-117.

69 J. Furustig, I. Dobryden, A. Almqvist, N. Almqvist and R. Larsson, Wear, 2016, 350-351, 74-81.

70 R. Bennewitz and J. T. Dickinson, MRS Bull., 2008, 33, 1174-1180.

71 O. Krivosheeva, M. Sababi, A. Dedinaite and P. M. Claesson, Langmuir, 2013, 29, 9551-9561.

72 F. Zhang, J. Pan and P. M. Claesson, Electrochim. Acta, 2011, 56, 1636-1645.

73 M. Sababi, F. Zhang, O. Krivosheeva, M. Forslund, J. Pan, P. M. Claesson and A. Dedinaite, J. Electrochem. Soc., 2012, 159, C364-C371.

74 C.-H. Chen, R. Hou, F. Zhang, S. Dong, P. M. Claesson, C. Lin and J. Pan, J. Electrochem. Soc., 2017, 164, C188-C193.

75 M. D'Acunto, F. Dinelli and P. Pingue, in Fundamentals of friction and wear at the nanoscale, ed. E. Gnecco and E. Meyer, Springer International Publishing, Switzerland, 2015.

76 R. G. Horn and J. N. Israelachvili, J. Chem. Phys., 1981, 75, 1400-1411.

77 L. Calabri, N. Pugno and S. Valeri, J. Phys.: Condens. Matter, 2008, 20, 474208.

78 D. Tranchida, S. Piccarolo and R. A. C. Deblieck, Meas. Sci. Technol., 2006, 17, 2630-2636.

79 A. A. Bukharaev, N. V. Berdunov, D. V. Ovchinnikov and K. M. Salikhov, Scanning Microsc., 1998, 12, 225-234. 